Publ. RIMS, Kyoto Univ.

21 (1985), 191-204

\title{
A Noncommutative Marcinkiewicz Theorem
}

By

\author{
Klaus BAUMANN and Gerhard C. HEGERFELDT ${ }^{*}$
}

\begin{abstract}
Abstroce
Many probabilistic notions and results can be carried over to noncommutative algebraic structures such as groups, $C^{*}$-algebras, Lie algebras and "tensor algebras. In this paper we consider a set $\mathscr{M}$ of (unbounded) operators in a Hilbert space with cyclic vector $\phi_{0}$. Generalized moments (' $n$-point functions') are defined by $\left\langle\phi_{0}, A_{1} \cdots A_{n} \phi_{0}\right\rangle, A_{i} \in \mathscr{M}$, and generalized cumulants ('truncated $n$-point functions') are defined in analogy to probability theory. The classical Marcinkiewicz Theorem states that if the characteristic function of a random variable $\xi$ is the exponential of a polynomial $P$, then $P$ has degree at most 2 and $\xi$ is normal ; i.e., if the cumulants of $\xi$ vanish for $n \geqq N$, some $N$, then they vanish for all $n>2$. In this latter form the result is generalized to the above non-commutative case. Robinson [11] proved an analogous result for quantum fields by using the Wightman axioms and the canonical commutation relations, respectively. In our result, however, only positivity of the scalar product enters. The structure of the operator set $\mathscr{M}$ is shown to be an extension of that of generalized free fields. Applications to representations of Lie algebras and Lie groups are given.
\end{abstract}

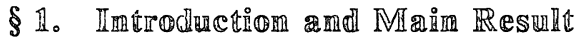

The extension of probabilistic notions and results to groups has been investigated for some time now (see e.g., [1], [2], [3]). The extension of such notions to $\mathbb{C}^{*}$ algebras has become an active area of research in the framework of the TomitaTakesaki theory (see, e.g., [4], [5]). Streater [6] introduced the notion of an infinitely divisible representation of Lie algebras and derived a criterion (see also [7]). A more general algebraic setting was considered by one of us [8].

Representations of *-tensor algebras, which arise in quantum field theory, and

Communicated by H. Araki, May 25, 1984.

* Institut für Theoretische Physik, Universität Göttingen, Göttingen, West Germany. 
associated states where investigated there, the notions of infinitely divisible and indecomposable states were introduced, and two important theorems of $\mathbb{K}$ hinchine $[9, \S 6.2]$ were generalized. All results of [8] carry over to free *-algebras generated by a set $\mathfrak{M}$. In a forthcoming paper, one of us [10] will prove a generalization of Cramer's Theorem and of the central limit theorem. The setting of the present paper is close to the latter and to [8] although no need arises to consider different representation. A fixed set $\mathscr{C l}$ of (unbounded) operators will do.

In its simplest form, the Marcinkiewicz Theorem states that if a positive definite function on $R$ is the exponential of a polynomial then this polynomial is of degree at most 2 [9]. An alternative formulation is that a random variable whose characteristic function $E(t)$ is the exponential of a polynomial has a normal or degenerate distribution.

Since the cumulants of a random variable are obtained from the derivatives of $\ln E(t)$ at $t=0$, the Marcinkiewicz Theorem can be formulated as follows :

Theorem (Marcinkiewicz). If the cumulants of a random variable $\xi$ vanish for all $n \geqq N$, some $N$, then they vanish for $n>2$, and thus $\xi$ is normal or degenerate.

In this form the theorem lends itself to a noncommutative generalization. A random variable $\xi$ is usually considered as a measurable function, with expectation given by integration with respect to a probability measure. But it can also be regarded as a multiplication operator in the corresponding $\mathrm{L}^{2}$-space. Different random variables commute as multiplication operators. The generalization now consists in allowing an arbitrary collection $\mathscr{M}$ of (possibly noncommuting and unbounded) operators defined on some invariant dense domain $\mathscr{D}$ in some Hilbert space. Expectation is replaced by $\left\langle\phi_{0}, \cdot \phi_{0}\right\rangle$ where $\phi_{0}$ is a unit vector in $\mathscr{D}$.

For $\mathscr{D}$ we take the smallest such domain, namely all polynomials in elements of $\mathscr{C}$ applied to $\phi_{0}$. We do not assume that the elements in $\mathscr{M}$ are self-adjoint or symmetric ; instead we assume that the set $\mathscr{M}$ is hermitian, i.e. $A \in \mathscr{M}$ implies

$$
A^{*}{ }_{\mid \mathscr{T}} \in \mathscr{M} \text {. }
$$

Without loss of generality we can assume that $\mathscr{M}$ is linear.

The moments of random variables, $\left\langle\xi_{1} \cdots \xi_{n}\right\rangle:=E \xi_{1} \cdots \xi_{n}$, are now replaced by 


$$
\left\langle A_{1} \cdots A_{n}\right\rangle:=\left\langle\phi_{0}, A_{1} \cdots A_{n} \phi_{0}\right\rangle, A_{i} \in \mathscr{e},
$$

where the order is in general important. The object in Eq. (1.1) will be called an npoint function or a (noncommutative) moment.

The analog of cumulants will be defined in Eq. (2.2). They are modeled after the usual cumulants and after the truncated $n$-point functions in quantum field theory. We will call them truncated $n$-point functions or (noncommutative) cumulants and denote them by $\left\langle A_{1} \cdots A_{n}\right\rangle_{T}$. The analog of a normal random variable is then a system of operators and corresponding $n$-point functions whose cumulants vanish for $n>2$ and are not identically zero for $n=2$. In quantum field theory this is known as a generalized free field.

The following main theorem uses only positivity in the form of the scalar product of a Hilbert space and does not need any further assumptions usually made in quantum field theory or in quantum mechanics.

Theorem (Noncommutative Marcinkiewicz Theorem): Let $\mathscr{M}$ be a hermitian set of operators and let $\phi_{0}$ be a unit vector in the domain of any product of operators in $\mathscr{M}$, with $n$-point functions (moments) $\left\langle\phi_{0}, A_{1} \cdots A_{n} \phi_{0}\right\rangle$. If all associated truncated n-point functions (cumulants) vanish for $n>N$, some $N$, then they vanish for $n>2$.

Remark. In quantum field theory an analogous result was first derived by Robinson[11] who reduced it to the commutative case by use of the Wightman axioms or canonical commutation relations, respectively. Using the spectral condition, Borchers [12] and, independently, one of us [13], showed that the vanishing for $n=2 N$, some $N>0$, was already sufficient. $\mathbb{A}$ similar result was obtained for a class of Euclidean fields by Newman[14].

As a by-product of our proof we obtain the structure of the operator set $\mathscr{C l}$ of the theorem, a structure which is well-known from generalized free fields.

Corollary. Let the assumptions of the theorem hold. Let $\mathscr{D}$ be obtained by acting on $\phi_{0}$ with all polynomials in elements of $\mathscr{M}$. Then for any $A \in \mathscr{M}$, any $\phi$ $\in \mathscr{D}$ is an entire vector for $A$; hence if $A$ is symmetric it is essentially self-adjoint on $\mathscr{D}$. In the sense of strong convergence of power series one has on $\mathscr{D}$ 


$$
e^{z_{1} A_{1}} e^{z_{2} A_{2}}=\exp \left\{\frac{z_{1} z_{2}}{2}\left\langle A_{1} A_{2}-A_{2} A_{1}\right\rangle\right\} e^{z_{1} A_{1}+z_{2} A_{2}}
$$

and the 'moment generating function' is given by

$$
\begin{aligned}
\left\langle\phi_{0},\right. & \left.e^{z_{1} A_{1} \ldots} e^{z_{n} A_{n}} \phi_{0}\right\rangle \\
& \left.=\exp \left\{\sum z_{i}\left\langle A_{i}\right\rangle+\frac{1}{2}\left\langle\left[\sum z_{i}\left(A_{i}-\left\langle A_{i}\right\rangle\right)\right]^{2}\right\rangle+\frac{1}{2} \sum_{i<j} z_{i} z_{j}<\left[A_{i}, A_{j}\right]\right\rangle\right\} .
\end{aligned}
$$

If $A, B \in \mathscr{M}$ then their commutator is a multiple of unity on $\mathscr{D}$.

Section 2 deals with the definition of truncations and simple combinatorics. In Section 3 we prove an estimate for $n$-point functions and convergence of the exponential series. In Section 4, Eqs. (1.2) and (1.3) are proved by means of Schwarz's inequality and by a generalized Liouville Theorem for entire functions.

In Section 5 we discuss applications to representation theory, in particular for Lie groups and Lie algebras.

\section{§ 2. Truncation Combinatorics}

We collect a few simple facts about noncommutative cumulants (truncated $n$ point functions).

Definition 2.1. Let $I$ be a set. A (finite) partition $P$ of $I$ is a collection $\left\{I_{k}\right\}$ of disjoint nonempty subsets of $I$ with $\cup I_{k}=I$. The set of all partitions of $I$ is denoted by $P_{I}$.

$\mathbb{D e}$ 俩inition 2.2. For each $i \in \mathbb{N}$, let $A_{i} \in \mathscr{M}$ be given. For each finite $I, I=\left\{i_{1}\right.$, $\left.\cdots, i_{l}\right\}, i_{1}<i_{2} \cdots<i_{l}$, we define

$$
A_{I}:=A_{i_{1}} \cdots A_{i_{l}} \text {. }
$$

Defimition 2.3. The cumulants $\langle\cdots\rangle_{T}$ are defined inductively as follows ${ }^{1)}[15]$.

$$
\left\langle A_{I}\right\rangle=\left\langle A_{I}\right\rangle_{T}+\sum_{\substack{P \in p_{I} \\ k>1}}\left\langle A_{I_{1}}\right\rangle_{T} \cdots\left\langle A_{I_{k}}\right\rangle_{T}
$$

1) Borchers [16] and Ruelle [17] use the notion of a partition as an ordered set. Then the expression for $\left\langle A_{I}\right\rangle_{T}$ looks different. By means of a product for states defined by $n$-point functions one obtains a very elcgant formalism in which the truncated state becomes a logarithm. See also [8]. 
Exæmples。 One has

$$
\begin{aligned}
\left\langle A_{1}\right\rangle= & \left\langle A_{1}\right\rangle_{T}, \\
\left\langle A_{1} A_{2}\right\rangle= & \left\langle A_{1} A_{2}\right\rangle_{T}+\left\langle A_{1}\right\rangle_{T}\left\langle A_{2}\right\rangle_{T}, \\
\left\langle A_{1} A_{2} A_{3}\right\rangle= & \left\langle A_{1} A_{2} A_{3}\right\rangle_{T}+\left\langle A_{1} A_{2}\right\rangle_{T}\left\langle A_{3}\right\rangle_{T}+\left\langle A_{1}\right\rangle_{T}\left\langle A_{2} A_{3}\right\rangle_{T} \\
& +\left\langle A_{1} A_{3}\right\rangle_{T}\left\langle A_{2}\right\rangle_{T}+\left\langle A_{1}\right\rangle_{T}\left\langle A_{2}\right\rangle_{T}\left\langle A_{3}\right\rangle_{T} .
\end{aligned}
$$

An immediate consequence is the following.

Lemma 2.1 Let $I=\{1, \cdots, n\}$. Then

$$
\left\langle A_{1} \cdots A_{n}\right\rangle=\left\langle A_{1} \cdots A_{n}\right\rangle_{T}+\sum_{\substack{n \in I^{\prime} \\ I^{\prime} \neq I}}\left\langle A_{I \backslash I^{\prime}}\right\rangle\left\langle A_{I^{\prime}}\right\rangle_{T} .
$$

Proof. In Eq. (2.2) one can assume that $n \in \mathbb{I}_{k}$. Putting $I^{\prime}=I_{k}$ and summing first over $P_{I \backslash I^{\prime}}$ in Eq. (2.2) yield $\left\langle A_{I \backslash I^{\prime}}\right\rangle\left\langle A_{I^{\prime}}\right\rangle_{T}$.

It is now easy to invert Eq. (2.2) by straight forward induction, using Eq. (2.3). This gives a well-known expression.

COrøllæry 2.1。 One has

$$
\left\langle A_{I}\right\rangle_{T}=\sum_{P \in P_{I}}(-1)^{k-1}(k-1) !\left\langle A_{I_{1}}\right\rangle \cdots\left\langle A_{I_{k}}\right\rangle
$$

We now state another expression which closely resembles the definition of cumulants in probability theory [9]. In the noncommutative case the order of factors is important. The logarithm below is defined by its Taylor series around 1 .

Proposition 2.1. In the sense of formal power series one has

$$
\left\langle A_{1} \cdots A_{n}\right\rangle_{T}=\frac{\partial}{\partial x_{1}} \cdots \frac{\partial}{\partial x_{n}} \ln \left\langle e^{x_{1} A_{1}} \cdots e^{x_{n} A_{n}}\right\rangle_{\left.\right|_{x=0}}
$$

This expression is generally accepted by the workers in the field, but we have not found a reference. A proof can be given by the following elementary fact.

Lemmm 2.2. Let $f$ be a (formal) power series in $x_{1}, \ldots, x_{N}, N<\infty$. Let $\partial_{J}=\prod_{j \in J} \frac{\partial}{\partial x_{j}}$, and let $I=\{1, \cdots, n\}$. Then

$$
\partial_{1} \cdots \partial_{n} e^{f}=\left(\partial_{1} \cdots \partial_{n} f\right) e^{f}+\sum_{\substack{n \in I^{\prime} \subset I \\ I^{\prime} \neq I}}\left(\partial_{I \backslash I^{\prime}} e^{f}\right) \partial_{I^{\prime}} f
$$


Proof. This holds for $n=1$. Let it hold for all $\nu, \nu \leqq n<N$. Using Eq. (2.6) for $J=\{2, \cdots, n+1\}$ we have

$$
\begin{gathered}
\partial_{1} \partial_{2} \cdots \partial_{n+1} e^{f}=\left(\partial_{1} \cdots \partial_{n+1} f\right) e^{f}+\left(\partial_{1} e^{f}\right) \partial_{2} \cdots \partial_{n+1} f \\
\quad+\sum_{n+J_{\substack{1 \\
J^{\prime} \neq J} J}\left\{\left(\partial_{\{1\} \cup J \backslash J^{\prime}} e^{f}\right) \partial_{J^{\prime}} f+\left(\partial_{J \backslash J^{\prime}} e^{f}\right) \partial_{\{1\} \cup J^{\prime}} f\right\}}
\end{gathered}
$$

which is Eq. (2.6) for $n+1$.

Proof of Proposition 2.1. In Eq. (2.6) we choose

$$
f=\ln \left\langle e^{x_{1} A_{1}} \cdots e^{x_{n} A_{n}}\right\rangle .
$$

Then

$$
\left.\partial_{1} \cdots \partial_{n} e^{f}\right|_{x=0}=\left\langle A_{1} \cdots A_{n}\right\rangle
$$

and Eq. (2.6), at $x=0$, is identical to Eq. (2.3) with $\left\langle A_{J}\right\rangle_{T}$ replaced by $\partial_{J} f_{\left.\right|_{x=0}}$. Since $\left\langle A_{k}\right\rangle=\left\langle A_{k}\right\rangle_{T}$ for fixed $k$ the statement follows by induction.

\section{$\S 3$. Convergence of Exponential Series}

We need the following estimate.

Lemma 3.1. Let all truncated $n$-point functions (cumulants) vanish for $n>$ $N \geqq 1$. Let $\mathscr{M}_{0} \subset \mathscr{M}$ be finite, and let

$$
M_{T}:=\max \left\{\left|\left\langle A_{1}^{\prime} \cdots A_{\nu}^{\prime}\right\rangle_{T}\right|^{1 / \nu}, A_{i}^{\prime} \in \mathscr{M}_{0}, 1 \leqq \nu \leqq N\right\}
$$

Then, for $A_{1}, \cdots, A_{m} \in \mathscr{M}_{0}$,

$$
\left|\left\langle A_{1} \cdots A_{m}\right\rangle\right| \leqq\left(2 M_{T}\right)^{m} \frac{(m-1) !}{(m-N)(m-2 N) \cdots(m-k N)}
$$

where $1 \leqq m-k N \leqq N$ for $m>N$ while for $m \leqq N$ the denominator is to be omitted.

Proof. For $m=1$, Eq. (3.1) holds true. Assume it holds true for all $\nu \leqq m-1$. Now we use Eq. (2.3) for $n=m$ and with $\left\langle A_{I}^{\prime}\right\rangle_{T}=0$ for $\left|I^{\prime}\right|>N$. The number of subsets of $\{1, \cdots, m\}$ of length $\nu$ which contain $m$ is $\left(\begin{array}{c}m-1 \\ \nu-1\end{array}\right)$. 
If $m>N$ we obtain, by induction hypothesis,

$$
\left|\left\langle A_{1} \cdots A_{m}\right\rangle\right| \leqq \sum_{\nu=1}^{N}\left(\begin{array}{c}
m-1 \\
\nu-1
\end{array}\right)\left(2 M_{T}\right)^{m-\nu} \frac{(m-\nu-1) !}{(m-\nu-N) \cdots} M_{T}^{\nu}
$$

We replace the denominator by $(m-2 N) \cdots$ if $m>2 N$ and by 1 otherwise. Since

$$
\frac{(m-1) !(m-\nu-1) !}{(\nu-1) !(m-\nu) !} \leqq \frac{(m-1) !}{(\nu-1) !(m-N)}
$$

we can continue the estimate with

$$
\leqq\left(2 M_{T}\right)^{m}(m-1) ! \frac{1}{2} \sum_{\nu=1}^{N} \frac{1}{(\nu-1) !} \frac{1}{2^{\nu-1}} \frac{1}{(m-N)(m-2 N) \cdots}
$$

Since the sum $\sum$ is less than $e^{1 / 2}$, Eq. (3.1) follows for $m>N$.

For $m \leqq N$ the estimate is similar, with the denominator now equal to 1 .

This estimate enables us to prove convergence of multiple power series for exponentials.

Theorem 3.1. Let all truncated $n$-point functions (cumulants) vanish for $n$ $>N$, some $N$. For $A_{1}, \cdots, A_{n} \in \mathscr{M}$ and any $\phi \in \mathscr{D}$, the multiple power series for

$$
e^{z_{1} A_{1} \ldots} e^{z_{n} A_{n}} \phi, \quad z_{i} \in \mathbb{C}
$$

converges absolutely. A fortiori, a symmetric $A \in \mathscr{C}$ is essentially selfadjoint on $\mathscr{D}$.

Proof. We can assume $\phi=B_{1} \ldots B_{k} \phi_{0}$. We take $\mathscr{M}_{0}=\left\{A_{1}, A_{1}^{*}, \cdots, B_{k}, B_{k}^{*}\right\}$. Let

$$
M_{\nu}:=\max \left\{\left|\left\langle A_{1}^{\prime} \cdots A_{\nu}^{\prime}\right\rangle\right|, A_{i}^{\prime} \in \mathscr{M}_{0}\right\}, \nu=1,2, \cdots
$$

We have

$$
\begin{gathered}
\sum_{l} \prod_{i=1} \frac{\left|z_{i}\right|^{l_{i}}}{l_{i} !}\left\|A_{1}^{l_{1}} \ldots A_{n}^{l_{n}} B_{1} \ldots B_{k} \phi_{0}\right\| \\
\leqq \sum_{i} \prod_{i} \frac{\left|z_{i}\right|^{l_{i}}}{l_{i} !} M_{2\left(l_{1}+\cdots l_{n}+k\right)}^{1 / 2} \\
\quad=\sum_{l=0}^{\infty} \frac{1}{l !} M_{2(l+k)}^{1 / 2}\left(\sum\left|z_{i}\right|\right)^{l}
\end{gathered}
$$


The root criterion for the convergence radius $R$, Hadamard's formula $1 / R=$ $\varlimsup_{l \rightarrow \infty}\left|a_{l}\right|^{1 / l}$, gives by Lemma 3.1

$$
\begin{aligned}
\frac{1}{R}= & \varlimsup_{l \rightarrow \infty}\left(2 M_{T}\right)^{1+\frac{k}{l}}\left\{\frac{(2 l+2 k-1) \cdots(l+1)}{l !}\right\}^{1 / 2 l} \\
& \cdot\{(2 l+2 k-N)(2 l+2 k-2 N) \cdots\}^{-1 / 2 l}
\end{aligned}
$$

By Stirling's formula one sees that the second factor is bounded and that the third factor goes to zero. Hence $R=\infty$.

Remark. This result shows that each $A \in \mathscr{M}$ is closable and that its closure, also denoted by $A$, contains in its domain all vectors of the form $e^{z_{1} A_{1}} \ldots e^{z_{n} A_{n}} \phi$, $\phi \in \mathscr{D}$. Hence $e^{z_{i} A_{i}}, i=1,2, \cdots$, define operators which can be multiplied on $\mathscr{D}$.

Corollary 3.1. Let all cumulants of order greater than $N$ vanish. Then for $A_{1}, \cdots, A_{n}$ there is a polynomial $P_{A_{1} \cdots A_{n}}\left(z_{1}, \cdots, z_{n}\right)$ of degree at most $N$ such that

$$
\left\langle e^{z_{1} A_{1}} \cdots e^{z_{n} A_{n}}\right\rangle=\exp \left\{P_{A_{1} \cdots A_{n}}\left(z_{1}, \cdots, z_{n}\right)\right\} \text {. }
$$

$P_{A_{1} \cdots A_{n}}$ is given by

$$
\sum_{|l| \leqq N} \frac{1}{l_{1} !} \cdots \frac{1}{l_{n} !}\left\langle A_{1}^{l_{1}} \cdots A_{n}^{l_{n}}\right\rangle_{T} z_{1}^{l_{1}} \cdots z_{n}^{l_{n}}
$$

Proof. The power series for the 1.h.s. of Eq. (3.5) converges absolutely. For $z=0$ it is 1 so that the Taylor series converges around 1 in a neighborhood of $z=0$. Hence Proposition 2.1 holds here for functions, not only formal power series. Since

$$
\begin{aligned}
\frac{\partial^{l_{1}}}{\partial z_{1}^{l_{1}}} & \cdots \frac{\partial^{l_{n}}}{\partial z_{n}^{l_{n}}} \ln \left\langle e^{z_{1} A_{n}} \cdots e^{z_{n} A_{n}}\right\rangle_{\left.\right|_{z=0}} \\
& =\frac{\partial}{\partial z_{11}} \cdots \frac{\partial}{\partial z_{1 l_{1}}} \frac{\partial}{\partial z_{21}} \cdots \ln \left\langle\left.\prod_{i=1}^{l_{1}} e^{z_{1 i} \cdot A_{1}} \ldots\right|_{z=0}\right.
\end{aligned}
$$

these derivatives are given by cumulants, which vanish for $l_{1}+\cdots+l_{n}>N$.

\section{§4. The Form of the Generating Function. Proof of Main Theorem}

We will need some simple applications of a generalized Liouville theorem [18]. This states that if $f(z)$ is entire and $\operatorname{Re} f(z) \leqq C|z|^{\rho}$ for large $|z|$ then $f(z)$ is a polynomial of degree at most $\rho$. 
Lemma 4 . $\mathbb{H}_{\circ}$ a) If $P(z)$ is a polynomial and

$$
\operatorname{Re} P(z) \leqq P(\operatorname{Re} z)
$$

then $P$ is of degree at most 2.

b) If $f\left(z_{1}, z_{2}\right)$ is entire and

$$
\operatorname{Re} f\left(z_{1}, z_{2}\right) \leqq C\left\{\left|z_{1}\right|^{2}+\left|z_{2}\right|^{2}+1\right\}
$$

then $f$ is a polynomial of degree at most 2 .

c) If $f\left(z_{1}, z_{2}, z_{3}\right)$ is entire and

$$
\operatorname{Re} f\left(z_{1}, z_{2}, z_{3}\right) \leqq\left\{\begin{array}{l}
C \cdot\left(\operatorname{Re} z_{1}\right)^{2}+C_{z_{3}} \cdot\left[\left(\operatorname{Re} z_{2}\right)^{2}+1\right] \\
C^{\prime} \cdot\left(\operatorname{Re} z_{3}\right)^{2}+C_{z_{1}}^{\prime} \cdot\left[\left(\operatorname{Re} z_{2}\right)^{2}+1\right]
\end{array}\right.
$$

where $C_{z_{3}}$ and $C_{z_{1}}^{\prime}$ depend on $z_{1}$ and $z_{3}$, respectively, then $f$ is a polynomial of degree at most 2.

Proof. a) $\mathbb{P}$ must have real coefficients and must be of even degree, $2 n$ say, with positive highest coefficient, unless it is of degree 1 . If the degree were $2 n, n \geqq$ 2 , then one would have with $z=t e^{i \phi}$

$$
t^{2 n}\left(\cos ^{2 n} \phi-\cos 2 n \phi\right) \geqq 0 \text {. }
$$

This is violated for $\phi=\pi / n$.

b) For fixed $z_{1}, z_{2}, f\left(z_{1} z, z_{2} z\right)$ is a polynomial of degree at most 2 . The coefficient of $z^{0}$ is $f(0,0)$ and that of $z^{1}$ is

$$
\frac{\partial f}{\left.\partial z\right|_{z=0}}=\left.\frac{\partial f}{\partial z_{1}}\right|_{z=0} z_{1}+\left.\frac{\partial f}{\partial z_{2}}\right|_{z=0} z_{2}
$$

which is linear in $z_{1}, z_{2}$. Similarly for $z^{2}$. Finally put $z=1$.

c) By part $b$ ), $f$ is a polynomial in $z_{1}, z_{2}$ of degree at most 2 for fixed $z_{3}$, and similarly for $z_{1}$ fixed. Thus $f$ is a polynomial in $z_{1}, z_{2}, z_{3}$ of degree at most 3 with the only possible third order term of the form $b z_{1} z_{2} z_{3}$ since $z_{1}^{2} z_{3}^{2}$ is ruled out by Eq. (4.2). The combination

$$
\begin{aligned}
& \frac{1}{4}\left\{f\left(z_{1}, z_{2}, z_{3}\right)+f\left(-z_{1},-z_{2}, z_{3}\right)+f\left(-z_{1}, z_{2},-z_{3}\right)+f\left(z_{1},-z_{2},-z_{3}\right)\right\} \\
& =: \hat{f}\left(z_{1}, z_{2}, z_{3}\right)
\end{aligned}
$$

has the form $\hat{f}=a_{0}+a_{1} z_{1}^{2}+a_{2} z_{2}^{2}+a_{3} z_{3}^{2}+b z_{1} z_{2} z_{3}$ and fulfills the inequality 


$$
\operatorname{Re} \hat{f}\left(z_{1}, z_{2}, z_{3}\right) \leqq C\left(\operatorname{Re} z_{1}\right)^{2}+\frac{1}{2}\left(C_{z_{3}}+C_{-z_{3}}\right)\left[\left(\operatorname{Re} z_{2}\right)^{2}+1\right]
$$

Assuming $b \neq 0$ we put $z_{1}=z_{2}=i y$ and $z_{3}=-\left(1+a_{1}+a_{2}\right) / b$. The above inequality gives

$$
\operatorname{Re}\left(a_{0}+y^{2}+a_{3}\left(1+a_{1}+a_{2}\right)^{2} / b^{2}\right) \leqq \widehat{C}
$$

for all $y \in \mathbb{R}$, which is a contradiction. Therefore $b=0$.

We can now determine the form of the polynomial $P_{A_{1} \cdots A n}$ in Eq. (3.5) for $n \leqq 3$ by using Schwarz's inequality.

We define the hermitian part $\mathscr{M}_{h}$ of $\mathscr{e l}^{\text {by }}$

$$
\mathscr{M}_{h}=\left\{A \in \mathscr{M} ; A^{*}{ }_{\mathscr{I}}=A\right\} .
$$

We can and will assume $\mathscr{A}$ to be linear. One has on $\mathscr{D}$

$$
A=\frac{1}{2}\left(A+A^{*}\right)+i\left(A-A^{*}\right) / 2 i
$$

so that $\mathscr{e l}^{=} \mathscr{e}_{h}+i \mathscr{M}_{h}$.

Lemma 4。2. For $n \leqq 3, P_{A_{1} \cdots A_{n}}$ has degree at most 2 .

Proof. We first let $A_{i} \in \mathscr{M}_{h}, i=1,2,3$. For $n=1$ we have by Theorem 3.1

$$
\left\langle\phi_{0}, e^{z A_{1}} \phi_{0}\right\rangle=\left\langle e^{z A_{1} / 2} \phi_{0}, e^{z A_{1} / 2} \phi_{0}\right\rangle \text {. }
$$

Schwarz's inequality gives

$$
\operatorname{Re} P_{A_{1}}(z) \leqq P_{A_{1}}(\operatorname{Re} z)
$$

and so Lemma 4.1a) applies.

For $n=2$ we have, by Theorem 3.1,

$$
\left\langle\phi_{0}, e^{z_{1} A_{1}} e^{z_{2} A_{2}} \phi_{0}\right\rangle=\left\langle e^{z_{1} A_{1}} \phi_{0}, e^{z_{2} A_{2}} \phi_{0}\right\rangle .
$$

Schwarz's inequality gives

$$
\operatorname{Re} P_{A_{1} A_{2}}\left(z_{1}, z_{2}\right) \leqq \frac{1}{2} P_{A_{1}}\left(2 \operatorname{Re} z_{1}\right)+\frac{1}{2} P_{A_{2}}\left(2 \operatorname{Re} z_{2}\right)
$$

Lemma $4.1 b$ ) now applies.

Case $n=3$ : For $z_{1}$ fixed, 


$$
\left\langle e^{z_{1} A_{1}} \phi_{0}, e^{z A_{2}} e^{z_{1} A_{1}} \phi_{0}\right\rangle
$$

is the exponential of a polynomial in $z$. If one replaces, in case $n=1, \phi_{0}$ by $e^{z_{1} A_{1}} \phi_{0}$ and $A_{1}$ by $A_{2}$, this implies that this polynomial has degree at most 2 .

We now have

$$
\begin{aligned}
\left\langle e^{z_{1} A_{1}} e^{z_{2} A_{2}} e^{z_{3} A_{3}}\right\rangle & =\left\langle e^{\bar{z}_{1} A_{1}} \phi_{0}, e^{z_{2} A_{2}} e^{z_{3} A_{3}} \phi_{0}\right\rangle \\
& =\left\langle e^{z_{2} A_{2}} e^{z_{1} A_{1}} \phi_{0}, e^{z_{3} A_{3}} \phi_{0}\right\rangle
\end{aligned}
$$

Applying Schwarz's inequality to the r.h. sides gives, together with the preceding remark, two inequalities for $\operatorname{Re} P_{A_{1} A_{2} A_{3}}\left(z_{1}, z_{2}, z_{3}\right)$ as in Eq. (4.2). This proves the case $n=3$.

To obtain the statement for general $A^{\prime}$ s, we consider first $A_{k}=A_{k}^{\prime}+\lambda_{k} A_{k}^{\prime \prime}, A_{k}^{\prime}$, $A_{k}^{\prime \prime} \in \mathscr{M}_{h}$ and $\lambda_{k} \in \mathbb{R}$. By Corollary 3.1 the coefficients of the polynomials $P_{A_{1} A_{2} A_{3}}$ are polynomials in $\lambda_{1}, \ldots, \lambda_{3}$ of degree at most 2 . By analyticity and $\lambda_{k} \rightarrow i$, the general case follows.

Corollary 4.1. For $A_{1}, A_{2} \in \mathscr{A}$ one has

$$
e^{A_{1}} e^{A_{2}} \phi_{0}=\exp \left\{+\frac{1}{2}\left\langle\left[A_{1}, A_{2}\right]\right\rangle\right\} e^{A_{1}+A_{2}} \phi_{0}
$$

Proof. We can assume $\left\langle A_{1}\right\rangle=\left\langle A_{2}\right\rangle=0$. First let $A_{1}, A_{2} \in \mathscr{M}_{h}$. A straightforward calculation, using Lemma 4.2 and Eq. (3.6), gives with $z_{k}=i t_{k}, t_{k} \in \mathbb{R}$,

$$
\left\|e^{z_{1} A_{1}} e^{z_{2} A_{2}} \phi_{0}-e^{+\frac{1}{2}<\left[A_{1} A_{2}\right]>z_{1} z_{2}} e^{z_{1} A_{1}+z_{2} A_{2}} \phi_{0}\right\|^{2}=0
$$

As in the preceding proof, analyticity then gives the general statement.

Proof of Main Theorem and of Corollary. Corollary 4.1 and straightforward induction on $n$ gives

$$
e^{z_{1} A_{1}} \cdots e^{z_{n} A_{n}} \phi_{0}=e^{+\frac{1}{2}<\left[A_{1}, A_{2}\right]>z_{1} z_{2}} e^{z_{1} A_{1}+z_{2} A_{2}} e^{z_{3} A_{3}} \cdots e^{z_{n} A_{n}} \phi_{0}
$$

Differentiating at $z_{3}=\cdots=z_{n}=0$, which is possible by analyticity, gives $\mathbb{E q}$. (4.3) with $\phi_{0}$ replaced by $A_{3} \ldots A_{n} \phi_{0}$. Eq. (4.3) together with Lemma 4.2 shows that $P_{A_{1} \cdots A_{n}}$ in Eq. (3.5) is of degree at most 2. Eq. (3.6) then shows that $\left\langle A_{1} \cdots A_{n}\right\rangle_{T}=0$ for $n>2$. 


\section{§ 5. Discussion. Connection with Representation Theory}

Under the assumptions of the main theorem one has for the commutator of $A_{1}$, $A_{2} \in \mathscr{A}$ on $\mathscr{D}$

$$
\left[A_{1}, A_{2}\right]=\left\langle A_{1} A_{2}-A_{2} A_{1}\right\rangle I=\left\langle A_{1} A_{2}-A_{2} A_{1}\right\rangle_{T} I .
$$

If we assume $\mathscr{C}$ to be linear, which we can without loss of generality, then it suffices to consider $\mathscr{M}_{h}=\left\{A \in \mathscr{M}, A=A^{*}{ }_{\mid 9}\right\}$. For $A_{1}, A_{2} \in \mathscr{M}_{h}$, Eq. (5.1) reads

$$
\left[A_{1}, A_{2}\right]=2 i \operatorname{Im}\left\langle A_{1} A_{2}\right\rangle_{T} I \text {. }
$$

If the 2-point function is real on $\mathscr{M}_{h}$, one is in the commutative case.

Conversely, given a set $\mathfrak{M}$ and a form

$$
\mathfrak{M} \ni a_{1}, a_{2} \mapsto\left(a_{1}, a_{2}\right) \in \mathbb{C}
$$

which becomes positive semi-definite after linear extension one obtains a representation $\Pi$ of the free *-algebra generated by $\mathfrak{M}$ with truncated $n$-point functions given for $n=2$ by

$$
\left\langle\Pi\left(a_{1}\right)^{*} \Pi\left(a_{2}\right)\right\rangle_{T}=\left(a_{1}, a_{2}\right)
$$

and zero otherwise. This is the same as in the construction of the generalized free quantum field.

The operators of the hermitian set $\mathscr{M}$ may originate from a representation of a Lie algebra with cyclic vector $\phi_{0}$. Then the vanishing of all higher cumulants places restrictions on the structure of the Lie algebra, and the representation can be integrated to a group representation.

For Lie groups our generalization of the Marcinkiewicz Theorem can be formulated as follows.

Theorem 5.1. Let $G$ be a finite-dimensional Lie group. Let $U$ be a unitary continuous representation of $G$ with cyclic vector $\phi_{0}$. If the generating function

$$
E(g)=\left\langle\phi_{0}, U(g) \phi_{0}\right\rangle
$$

is the exponential of a polynomial in some local coordinates in a neighbourhood of the group identity, then this polynomial is of degree at most 2 and $G$ is homomorphic to a direct product of an abelian Lie group and a Weyl group. 
A Weyl group is associated with the usual canonical commutation relations

$$
\left[Q_{j}, P_{k}\right]=i \delta_{j k}
$$

of quantum mechanics [19], [20]. By Eq. (5.2) one can introduce a suitable basis in the (now finite-dimensional) linear space $\mathscr{M}_{h}$ where part of the basis operators span the center of $\mathscr{M}_{h}$ and the remaining ones satisfy Eq. (5.3).

\section{Refieremees}

[1] Streater, R.F.: Current Commutation Relations, Continuous Tensor Products and Infinitely Divisible Group Representations. in: International School of Physics 'Enrico Fermi', Course XLV, R. Jost Ed., Academic Press, New York (1969), 247.

[2] Parthasarathy, K.R., Schmidt, K.: Positive Definite Kernels, Continuous Tensor Products, and Central Limit Theorems of Probability Theory. Lecture Notes in Mathematics 272, Springer-Verlag, Heidelberg, 1972.

[3] Heyer, H. : Probability Measures on Locally Compact Groups. Springer-Verlag, Berlin, 1977.

[4] Takesaki, M.: Theory of Operator Algebras I. Springer-Verlag, New York, 1979.

[5] Pedersen, G.K.: $C^{*}$-Algebras and their Automorphism Groups. Academic Press, London, 1979.

[6] Streater, R.F.: Infinitely divisible representations of Lie algebras. Z. Wahrscheinlichkeitstheorie verw. Geb. 19 (1971), 67-80.

[7] Mathan, D., Streater, R.F.: Infinitely divisible representations of Clifford algebras. $Z$. Wahrscheinlichkeitstheorie verw. Geb. 20 (1971), 308-316.

[8] Hegerfeldt, G.C.: Prime Field Decomposition and Infinitely Divisible States on Borchers' Tensor Algebra. Commun. math. Phys. 45 (1975), 137-152.

[9] Lukacs, E. : Characteristic Functions. $2^{\text {nd }}$ Edition, Griffin, London, 1970.

[10] Hegerfeldt, G.C. : to be published.

[11] Robinson, D.W.: A Theorem Concerning the Positive Metric. Commun. math. Phys. $\mathbb{1}$ (1965), 89-94.

[12] Borchers, H.J. : unpublished, quoted in [8], footnote 14.

[13] Baumann, K.: When is a Field Theory a Generalized Free Field ? Commun. math. Phys. 43 (1975), 221-223.

[14] Newman, C.M. : Inequalities for Ising Models and Field Theories which Obey the Lee-Yang Theorem. Commun. math. Phys. 1 (1975), 1-9.

[15] Haag, R.: Quantum Field Theories with Composite Particles and Asymptotic Conditions. Phys. Rev. $\mathbb{1} \mathbb{1 2}$ (1958), 669-673.

[16] Borchers, H.J. : Algebraic aspects of Wightman field theory. In : Sen, R.N., Weil, C. (Eds.) : Statistical mechanics and field theory. Haifa Lectures 1971 Halsted Press, New York, 1972.

[17] Ruelle, D. : Staíistical Mechanics. Benjamin, New York, (1969), 86. 
[18] Boas, R.P.: Entire Functions. Academic Press, New York, (1954), 3.

[19] Segal, I.E. : Mathematical Problems of Relativistic Physics. AMS, Providence RI, 1963.

[20] Putnam, C.R.: Commutation Properties of Hilbert Space Operators and Related Topics. Springer-Verlag, Berlin, 1967. 